\title{
ANCA positive crescentic glomerulonephritis outcome in a Central East European cohort: a retrospective study
}

\author{
Iuliana Andreiana ${ }^{1,2^{*}}$, Simona Stancu ${ }^{1,2}$, Andreea Avram ${ }^{1}$, Ludmila Taran ${ }^{1}$ and Gabriel Mircescu ${ }^{1,2}$
}

\begin{abstract}
Background: The recently suggested distinct pathogenic pathways for myeloperoxidase (MPO) and proteinase 3 (PR3) anti-neutrophilic cytoplasmic antibodies (ANCA) associated vasculitis could result in different modes of presentation and outcome. Moreover, kidney outcome was related to a new histopathologic classification of pauci-immune glomerulonephritis. As reports were not always concordant, possible because differences in severity of organ lesions and ethnicity, we evaluated the outcome of a cohort of Central-East European patients with crescentic glomerulonephritis in relation with ANCA specificity and histopathological classification.

Methods: Seventy-five patients were consecutively diagnosed by kidney biopsy (76\% MPO-ANCA specificity, $52 \%$ crescentic) and followed for a median period of 3.2 years. Study end-points were response to therapy, end stage renal disease (ESRD) and death.

Results: PR3-ANCA patients were younger, in higher proportion male and had higher Birmingham Vasculitis Activity Scores (BVAS). The kidney disease was severe at presentation (median creatinine $5 \mathrm{mg} / \mathrm{dL} ; 27 \%$ required temporary dialysis) and worst in PR3-ANCA positive patients (50\% patients needed temporary dialysis vs. $19 \%$ ). The lung was the second most affected organ (31 \% severe lung hemorrhage). Lung and kidney damage were related; the odds of hemorrhagic alveolitis in patients needing dialysis at presentation were 4 (95\% Cl 1-13; $p=0.006$ ) times higher than in those who did not. The rate of response to therapy (without signs of active vasculitis and stable or declining serum creatinine) was $60 \%$ and was associated with dialysis independency, older age and higher platelet number at presentation. The probabilities to survival 1 and 5 years for kidney and patient were 93 and $64 \%$, and respectively 88 and $67 \%$. Kidney survival was predicted by response to therapy and dialysis independency at presentation. Patients with BVAS $<15$ and responding to induction therapy had better chances of survival. Neither response to therapy nor outcome was influenced by ANCA specificity or by the histopathological class.
\end{abstract}

Conclusions: When kidney damage is severe in ANCA vasculitis, the need of dialysis at presentation and the response to induction therapy overcome the prognostic utility of both ANCA specificity and histopathological class.

Keywords: ANCA, Outcome, Pauci-immune glomerulonephritis, Histopathological classes

\footnotetext{
* Correspondence: iuliandreiana@yahoo.com

'Department of Nephrology and Dialysis, "Dr. Carol Davila" Teaching Hospital of Nephrology, 4th Calea Grivitei Street, Bucharest 010731, Romania

2Department of Internal Medicine and Nephrology, "Carol Davila" University of Medicine and Pharmacy, Bucharest, Romania
} 


\section{Background}

ANCA-associated vasculitis is a rare, but severe autoimmune disease [1-3]. Kidney involvement (i.e. pauciimmune, crescentic glomerulonephritis), is frequent in ANCA vasculitis $[4,5]$ and can cause acute renal injury, which is potentially fatal, mostly when associated with pulmonary hemorrhage $[6,7]$. Accordingly, patients seen in a nephrology setting may have distinct clinical features from those seen by rheumatologists or pneumologists and they raise complex therapeutic problems. The relatively few reported series which addressed patients with severe kidney disease suggested a poorer outcome in this set of patients $[1,2,8,9]$. Whether ANCA type is related to the severity of vasculitis in patients seen by nephrologists is a matter of debate $[1,2,4]$. Recent data suggest distinct pathogenic pathways for myeloperoxidase (MPO) and proteinase 3 (PR3) ANCA vasculitis, which could result in different modes of presentation and outcome, although overlapping features are not infrequent [10]. However, reports from different groups are not always concordant, possibly because of differences in ethnicity (PR3-ANCA is rare in Asians and in African Americans, where MPO-ANCA is frequent) and in geographical distribution (in Europe there is a North to South gradient of incidence in PR3 and a South to North gradient in MPO) [11-14]. Thus, the potential differences in phenotype between MPO and PR3-ANCA are still to be defined [10].

The histological features of kidney biopsy could have a certain relationship to the outcome. Older studies have suggested that the proportion of normal glomeruli was a predictor of a good renal outcome [15], while the inflammatory lesions [16], i.e. the proportion of crescents, predicted the response to immunosuppressive therapy and the proportion of sclerotic glomeruli was related to a worse renal prognosis $[17,18]$. More recently, a panel of international renal pathologists proposed and validated the first classification for ANCA vasculitis: patients in the sclerotic class ( $\geq 50 \%$ sclerotic glomeruli) had the worst renal prognosis, followed in order by those in the mixed ( $<50 \%$ normal, $<50 \%$ crescents, $<50 \%$ sclerotic), crescentic ( $\geq 50 \%$ crescents) and focal ( $\leq 50 \%$ crescents) class [19]. The prognostic utility of this classification was confirmed in some but not all subsequent studies [20-23].

We aimed to evaluate ANCA phenotypes, kidney and patient outcome in a cohort of Central-East European patients with severe crescentic glomerulonephritis admitted in a tertiary Nephrology center in relation with ANCA specificity and histopathological classification.

\section{Methods}

\section{Patients}

This is a retrospective cohort study on all adult patients consecutively diagnosed by kidney biopsy with pauci-immune crescentic glomerulonephritis and followed in a single Nephrology center serving a population of about 4.85 million. From January 2000 till January 2014, pauci-immune crescentic glomerulonephritis were diagnosed in 104 patients. Thirteen patients were excluded because they were ANCA negative, 4 because of incomplete data and 12 were lost to follow-up.

The final cohort comprised 75 patients with ANCA positive pauci-immune crescentic glomerulonephritis who were followed over a median period of $3.2[0.1$; 5.5] years.

All subjects signed an informed consent form authorising us to use their demographic and medical data in this study.

The study was performed in accordance with Helsinky Declaration and it was approved by the local Ethics Committee of the "Dr. Carol Davila" Teaching Hospital of Nephrology.

\section{Diagnosis and follow-up}

The criteria for pauci-immune crescentic glomerulonephritis diagnosis were crescents (cellular and/or fibrous) in at least $50 \%$ of examined glomeruli by light microscopy and a direct immunofluorescence assay of 0 to $1+$ on a scale from 0 to $4+[24]$. For this study, all the biopsies were reviewed by our pathologist and they were classified in four classes as proposed by Berden et al.: crescentic ( $\geq 50 \%$ glomeruli with cellular crescents), focal $(\geq 50 \%$ normal glomeruli), sclerotic ( $\geq 50 \%$ sclerotic glomeruli) or mixed ( $<50 \%$ normal glomeruli, $<50 \%$ glomeruli with cellular crescents, $<50 \%$ sclerotic glomeruli ) [19].

ANCAs were assessed by capture PR3-ANCA and MPO ANCA ELISA (Euroimmun ${ }^{\mathrm{Ts}}$, Lübeck, Germany) or by indirect immunofluorescence (monoclonal mouse antihuman myeloperoxidase antibodies Dako ${ }^{\mathrm{Tm}}$, Glostrup, Denmark).

Markers of kidney damage were proteinuria measured in $24 \mathrm{~h}$ urine collection, hematuria (macroscopic and microscopic, red blood cells casts), serum creatinine and the histopathologic classes.

Hemorrhagic alveolitis was diagnosed based on chest radiographs and acute anemia without evidence of another external bleeding, and was classified as severe when oxygen partial pressure in arterial blood decreased under $60 \mathrm{mmHg}$. Severe anemia was defined as hemoglobin level less than $7 \mathrm{~g} / \mathrm{dL}$.

Inflammation was assessed using erythrocyte sedimentation rate (ESR), serum fibrinogen, serum albumin, platelet count and white blood cells count.

The measurements were performed by standard laboratory methods on an Olympus AU 400 chemistry auto-analyzer, and a MINDRAY BC 3000 hematology auto-analyzer.

The severity of vasculitis was evaluated using Birmingham Vasculitis Activity Score ver. 3 (BVAS) [25]. 
The follow-up protocol included monthly visits with clinical (BVAS) and laboratory evaluation, until remission and every 3 months thereafter.
Response to therapy was defined as disappearance of hematuria, stable or improving serum creatinine and no signs of activity in other organs [26].

Table 1 Patients' characteristics at presentation

\begin{tabular}{|c|c|c|c|c|}
\hline Parameter & $\begin{array}{l}\text { All } \\
n=75\end{array}$ & $\begin{array}{l}\text { PR3-ANCA } \\
n=18\end{array}$ & $\begin{array}{l}\text { MPO-ANCA } \\
n=57\end{array}$ & $P$ value \\
\hline \multicolumn{5}{|l|}{ General } \\
\hline Gender (\% Male) & 48 & 78 & 39 & 0.004 \\
\hline Age $>65$ years $(\%)$ & 35 & 11 & 42 & 0.02 \\
\hline Age (years) & $60[53 ; 68]$ & $58.0[43 ; 62]$ & $63[55 ; 69]$ & 0.01 \\
\hline Cold season at diagnosis & $60 \%$ & $65 \%$ & $62 \%$ & 0.81 \\
\hline Time to diagnosis (mo) & $2.0[1.0 ; 5.6]$ & $1.5[1.0 ; 3.0]$ & $2.0[1.0 ; 6.0]$ & 0.12 \\
\hline \multicolumn{5}{|l|}{ Vasculitis symptoms } \\
\hline Constitutional (\%) & 84 & 94 & 81 & 0.17 \\
\hline Skin (\%) & 9 & 17 & 7 & 0.22 \\
\hline Ocular (\%) & 3 & 11 & 0 & 0.01 \\
\hline Upper respiratory (\%) & 8 & 22 & 4 & 0.01 \\
\hline Lung (\%) & 48 & 50 & 47 & 0.85 \\
\hline Severe lung hemorrhage & $31 \%$ & $39 \%$ & $28 \%$ & 0.39 \\
\hline Kidney (\%) & 100 & 100 & 100 & 1.00 \\
\hline Digestive (\%) & 4 & 0 & 5 & 0.32 \\
\hline CNS (\%) & 4 & 0 & 5 & 0.32 \\
\hline Organs affected (No) & $2[1 ; 2]$ & $2[1 ; 3]$ & $2[1 ; 2]$ & 0.08 \\
\hline BVAS & $17[15 ; 21]$ & $21[15 ; 22]$ & $17[15 ; 20]$ & 0.05 \\
\hline \multicolumn{5}{|l|}{ Inflammation } \\
\hline Hemoglobin (g/dL) & $8.5[7.5 ; 9.8]$ & $7.7[5.7 ; 9.5]$ & $9.0[7.8 ; 9.8]$ & 0.04 \\
\hline Severe anemia (\%) & 35 & 56 & 28 & 0.03 \\
\hline WBC $\left(\right.$ per $\mathrm{mm}^{3}$ ) & 9750 [7800; 14400] & 10700 [7800; 15075] & 9650 [7683; 13900] & 0.64 \\
\hline $\mathrm{ESR}(\mathrm{mm} / 1 \mathrm{~h})$ & $97[64 ; 120]$ & $100[80 ; 140]$ & $94[63 ; 120]$ & 0.18 \\
\hline Fibrinogen (mg/dL) & $680[568 ; 800]$ & $714[608 ; 806]$ & $670[547 ; 800]$ & 0.48 \\
\hline Serum albumin (mg/dL) & $3.6[3.10 ; 4.0]$ & $3.3[2.9 ; 3.7]$ & $3.7[3.1 ; 4.0]$ & 0.15 \\
\hline \multicolumn{5}{|l|}{ Kidney disease } \\
\hline \multicolumn{5}{|l|}{ Histopathologic class } \\
\hline Crescentic (\%) & 52 & 67 & 47 & 0.52 \\
\hline Focal (\%) & 5 & 6 & 5 & \\
\hline Mixed (\%) & 32 & 22 & 35 & \\
\hline Sclerotic (\%) & 11 & 6 & 12 & \\
\hline Creatinine (mg/dL) & $5.0[3.4 ; 7.9]$ & $7.2[4.2 ; 10.0]$ & $4.8[3.1 ; 7.2]$ & 0.04 \\
\hline Proteinuria (g/day) & $0.8[0.5 ; 2.2]$ & $1.0[0.4 ; 1.4]$ & $0.8[0.5 ; 2.3]$ & 0.73 \\
\hline Hematuria (per mm³) & $260[190 ; 800]$ & $535[288 ; 1200]$ & $230[183 ; 623]$ & 0.005 \\
\hline Macroscopic hematuria & $44 \%$ & $78 \%$ & $33 \%$ & 0.001 \\
\hline RBC casts (\%) & 37 & 72 & 28 & 0.02 \\
\hline Dialysis at presentation & $27 \%$ & $50 \%$ & $19 \%$ & 0.01 \\
\hline
\end{tabular}

Values are median [quartile 1; quartile 3] if not otherwise specified mo months, CNS Central nervous system, No number, BVAS Birmingham Vasculitis Activity Score, WBC white blood cells, ESR erythrocyte sedimentation rate, $R B C$ red blood cells 
Dialysis therapy was considered temporary when needed for less than 3 months.

Study end-points were response to therapy, end stage renal disease (ESRD) and death.

\section{Therapy}

Induction therapy was done with steroids ( 3 daily intravenous pulses with methylprednisolone $0.5-1 \mathrm{~g}$ then prednisone $0.5 \mathrm{mg} / \mathrm{kg}$ daily, gradually tapered after one month) associated with cyclophosphamide $0.5 \mathrm{~g} / \mathrm{m}^{2}$, two pulses in the first month, then one pulse monthly for the next five months in the majority of patients (the mean cumulative cyclophosphamide dose was $4.6 \mathrm{~g}$ ). Maintenance therapy consisted in prednisone (10 mg daily in the first year and $7.5 \mathrm{mg}$ daily afterwards) and azathioprine $1.5-2 \mathrm{mg} / \mathrm{kg}$ per day for at least 2 years. Plasmapheresis was only occasionally performed (5 patients), usually in case of severe hemorrhagic alveolitis, because this therapy is not reimbursed by the health insurance authority.

\section{Statistical analysis}

Categorical variables are presented as percentages and comparison tests were performed using Pearson $\chi^{2}$ test. Continuous variables are displayed as mean with $95 \%$ confidence interval $(95 \% \mathrm{CI})$ or median and quartiles $[1 ; 3]$, according to their distribution. Comparisons were done with ANOVA, Mann-Whitney and Kruskal-Wallis tests, as appropriate.

Survival analyses were conducted with the Kaplan-Meier method and Mantel-Cox log-rank test for comparisons. Models of binomial or multinomial logistic regression were built to estimate differences between groups at presentation. Multivariate Cox proportional hazard ratios (PHR) models were used to evaluate parameters associated with various outcomes.

A $p \leq 0.05$ was considered statistically significant.

Statistical analyses were performed with SPSS (SPSS Inc., Chicago, IL) and Analyse-it ${ }^{\text {tix }}$ (Analyse-it Software, Ltd., Leeds, UK) packages.

\section{Results}

\section{Patients' characteristics at presentation}

Table 1 shows the baseline characteristics of patients. The clinical condition at presentation was severe (median BVAS 17). All patients had severe kidney disease (median creatinine $5 \mathrm{mg} / \mathrm{dL}, 27 \%$ needed dialysis). The lung was the second most affected organ; $31 \%$ of patients had severe hemorrhagic alveolitis. The odds of hemorrhagic alveolitis in patients needing dialysis at presentation were 4 (95\% CI 1-13; $p=0.006)$ times higher than in those who did not.

Most patients (76 \%) were MPO-ANCA positive. PR3ANCA patients were younger, in higher proportion male, had higher BVAS, resulting from more frequent upper respiratory tract and eye symptoms and more severe kidney damage (Table 1, Fig. 1).

Most of the patients (52\%) were in the crescentic histopathologic class. PR3-ANCA and MPO-ANCA specificities had a similar distribution in the histopathologic classes (Table 1).

When evaluated by logistic regression, in patients with creatinine $\geq 5 \mathrm{mg} / \mathrm{dL}$, PR3-ANCA was associated with male gender and dialysis at presentation (Table 2).

\section{Response to therapy}

Forty-five patients (60\%) responded to the induction therapy in $4[2 ; 6]$ months. Neither the histopathologic class nor the ANCA specificity influenced the rate of response.

In multivariate Cox regression analysis, although older patients and those with higher platelets number responded better, the need for dialysis at presentation was the most powerful predictor of response: the hazard ratio of response was two and a half times higher in patients not needing dialysis at presentation (Table 3).

\section{Kidney outcome}

Of the 51 patients alive at the end of the observation period, 38 (51\% of the whole cohort) were dialysis-free.
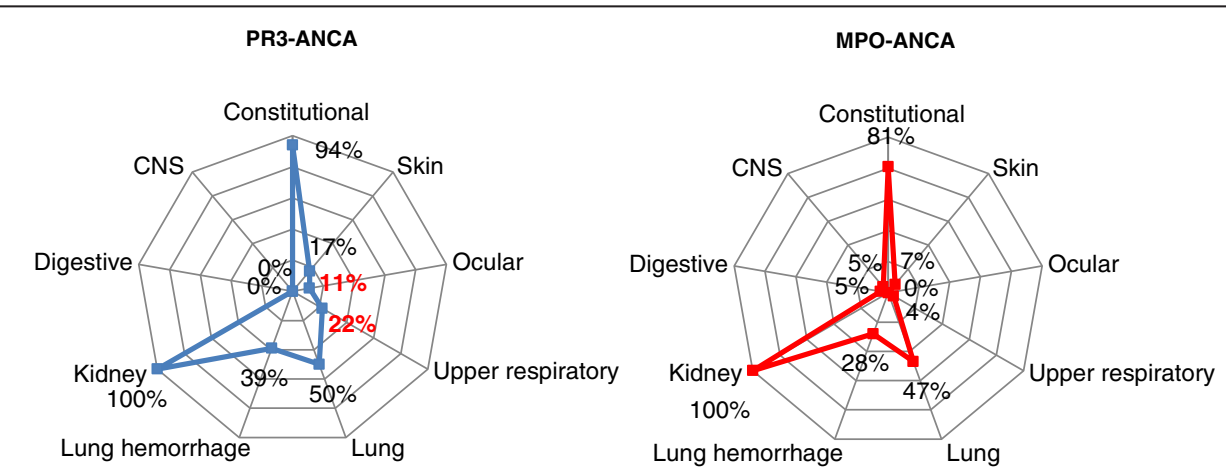

Fig. 1 Prevalence of organs affected according to ANCA serology. Legend: numbers represent percent from 75 patients which presented with the mentioned type of vasculitis manifestation; percents in red means statistically significant difference $(p<0.05)$. CNS Central nervous system 
Table 2 Differences in clinical presentation according to ANCA serology

\begin{tabular}{lllllll}
\hline Variables in equation $^{a}$ & B & S.E. & Exp(B) & $95 \% \mathrm{Cl}$ & Sig. \\
\hline Gender (Male) & 2.92 & 1.20 & 18.61 & 1.76 & 196.91 & 0.02 \\
Dialysis at presentation (Yes) & 2.10 & 0.92 & 8.16 & 1.34 & 49.56 & 0.02 \\
Constant & -0.93 & 0.68 & 0.40 & & & 0.17
\end{tabular}

${ }^{\mathrm{a}}$ Selection variable: Creatinine $\geq 5 \mathrm{mg} / \mathrm{dL}$

${ }^{\mathrm{b}}$ Cox \& Snell R square 0.46 ; Hosmer \& Lemeshow Goodness of fit $p=0.99$

Reference category is MPO-ANCA

As compared to dialysis dependent-patients, those dialysis-free were older females and had less severe kidney disease at presentation (lower creatinine levels, lower proteinuria and hematuria). The kidney survival was not influenced by the vasculitis severity, ANCA serology or the histopathological class (Table 4).

The unadjusted probability of kidney survival 1 and 5 years was $93 \%$ and $64 \%$. The median ESRD-free survival time was longer in responders to induction therapy and in those who did not need dialysis at presentation (Fig. 2). In a Cox PHR model, the response to induction therapy and dialysis at presentation were retained as independent predictors of kidney survival. The responders had thirty four times more chances to avoid ESRD ( $\mathrm{HR}=33.88,95 \% \mathrm{CI}$ 9.13-125.55, $p<0.001$ ), while those not needing dialysis at presentation had a $84 \%$ lower risk of kidney death $(\mathrm{HR}=0.16,95 \%$ CI 0.05-0.48, $p=0.001)$.

\section{Patient outcome}

Fifty one (68\%) patients survived. The main cause of death was pulmonary hemorrhage $(n=9 ; 38 \%$ of deaths).

Surviving patients had less severe vasculitis (lower BVAS), less severe kidney disease (lower creatinine and hematuria, and fewer needed dialysis at presentation) and higher serum albumin levels. They responded in higher proportion to induction therapy. ANCA specificity and the histopathological class did not influence the patient outcome (Table 5).

The median survival was 7.2 years, and the cumulative survival 1 and 5 years was $88 \%$ and $67 \%$. In a Cox PHR model, the independent predictors of death were a

Table 3 Predictors of the response to therapy; Multivariate Cox proportional hazards regression analysis

\begin{tabular}{lllllll}
\hline Variables & B & S.E. & Exp. (B) & $95 \%$ & Cl & Sig.* \\
\hline Age $>65$ years (No) & -0.87 & 0.35 & 0.42 & 0.21 & 0.84 & 0.01 \\
Ln (Platelets) & 1.36 & 0.51 & 3.91 & 1.44 & 10.59 & 0.01 \\
Albumin & 0.78 & 0.40 & 2.18 & 0.99 & 4.77 & 0.05 \\
Dialysis at presentation (No) & 0.91 & 0.52 & 2.47 & 0.89 & 6.87 & $<0.0001$ \\
\hline
\end{tabular}

$B$ coefficient b, S.E. standard error of coefficient b, Exp. (B) hazard ratio, $\mathrm{Cl}$ confidence interval, $\mathrm{Ln}$ logarithm

${ }^{*} p \leq 0.01$ statistically significant
Table 4 Patients' characteristics according to kidney outcome

\begin{tabular}{|c|c|c|c|}
\hline \multirow[t]{2}{*}{ Parameter } & \multicolumn{2}{|c|}{ End stage renal disease } & \multirow[t]{2}{*}{$P$ value } \\
\hline & No & Yes & \\
\hline Patients number & 38 & 13 & \\
\hline \multicolumn{4}{|l|}{ General } \\
\hline Gender (\% Male) & 47 & 69 & 0.01 \\
\hline Age (years) & $63[53 ; 69]$ & $51[35 ; 57]$ & 0.01 \\
\hline \multicolumn{4}{|l|}{ Vasculitis } \\
\hline PR3-ANCA (\%) & 64 & 36 & 0.37 \\
\hline MPO-ANCA (\%) & 78 & 22 & \\
\hline Severe lung hemorrhage (\%) & 24 & 39 & 0.30 \\
\hline BVAS & $15[13 ; 21]$ & $16[15 ; 21]$ & 0.63 \\
\hline $\begin{array}{l}\text { Response to induction } \\
\text { therapy }\end{array}$ & $97 \%$ & $15 \%$ & $<0.0001$ \\
\hline \multicolumn{4}{|l|}{ Inflammation } \\
\hline Hemoglobin (g/dL) & $9.0[7.8 ; 10.0]$ & $8.0[6.0 ; 9.0]$ & 0.07 \\
\hline WBC $($ per mm³) & $\begin{array}{l}9700[7800 ; \\
14200]\end{array}$ & $\begin{array}{l}8000[6300 ; \\
12500]\end{array}$ & 0.17 \\
\hline $\mathrm{ESR}(\mathrm{mm} / 1 \mathrm{~h})$ & $99[65 ; 115]$ & $110[82 ; 140]$ & 0.28 \\
\hline Fibrinogen (mg/dL) & $717[578 ; 800]$ & $720[612 ; 820]$ & 0.97 \\
\hline Albumin (g/dL) & $3.8[3.2 ; 4.0]$ & $3.5[3.1 ; 3.9]$ & 0.25 \\
\hline \multicolumn{4}{|l|}{ Kidney } \\
\hline \multicolumn{4}{|l|}{ Histopathologic class } \\
\hline Crescentic (\%) & 46 & 67 & 0.28 \\
\hline Focal (\%) & 7 & 0 & \\
\hline Mixed (\%) & 33 & 29 & \\
\hline Sclerotic (\%) & 13 & 5 & \\
\hline Creatinine (mg/dL) & $4.0[2.9 ; 5.8]$ & $9.0[4.5 ; 12.0]$ & 0.006 \\
\hline Proteinuria (g/day) & $0.6[0.4 ; 1.5]$ & $2.3[1.2 ; 3.0]$ & 0.007 \\
\hline Hematuria (per mm³) & 210 [150; 290] & 460 [230; 980] & 0.003 \\
\hline Dialysis at presentation (\%) & 8 & 62 & $<0.0001$ \\
\hline
\end{tabular}

Values are median [quartile 1; quartile 3] if not otherwise specified PR3-ANCA patients positive for anti-proteinase 3 antibodies, MPO-ANCA patients positive for anti-myeloperoxidase antibodies, BVAS Birmingham Vasculitis Activity Score, WBC white blood cells, ESR erythrocyte sedimentation rate

higher BVAS - for each increase in BVAS with three units, the risk of death was six times higher $(\mathrm{HR}=5.88$, $95 \%$ CI 1.03-33.58, $p=0.05$ ) - and the response to induction therapy - non-responders had five times less chances of survival $(\mathrm{HR}=0.20,95 \% \mathrm{CI} 0.07-0.53$, $p=0.0001$ ) (Fig. 3).

\section{Discussion}

This study reports the outcome in a cohort of Central East European patients with pauci-immune crescentic glomerulonephritis, where MPO-ANCA specificity was predominant, and the kidney damage severe and associated with lung hemorrhage. The patient and kidney outcomes were mainly influenced by the severity of kidney 

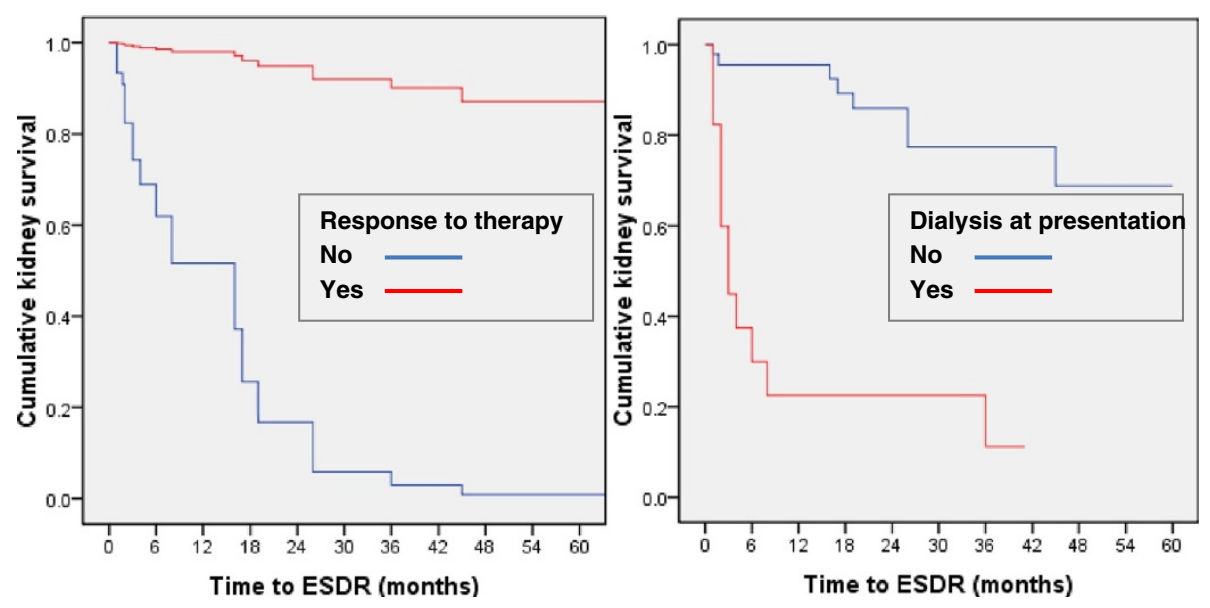

Fig. 2 Adjusted cumulative kidney survival according to the response to induction therapy (left) and to the need of dialysis at presentation (right). Legend: The median ESRD-free survival time was longer in responders to induction therapy [4.5 (4.1-4.9) vs. 1.0 (0.3-1.6) years; $p<0.0001]$ and in those who did not need dialysis at presentation [4.1 (3.6-4.7) vs. $0.9(0.3-1.6)$ years; $p<0.0001]$. ESRD End stage renal disease

damage at presentation and by the response to induction therapy, but not by ANCA specificity, suggesting that the severity of organ lesions could overcome the ANCArelated phenotypic differences. Moreover, the severity of kidney lesions influenced the patients' distribution in the histopathological classes, which probably limited its prognostic utility. In our cohort, median age was 60 years and gender distribution was balanced, as was reported in other series $[1-3,11,18,27-30]$. The majority of cases were diagnosed in cold seasons, which suggest that upper respiratory tract infections were triggers of autoimmunity [31, 32]. The median lag between first symptoms and diagnosis was 2 months, highlighting the difficulty to recognize these protean diseases, in line with other reports $[1,29]$.

The main feature of this cohort was severe kidney disease (median creatinine $5 \mathrm{mg} / \mathrm{dL} ; 27 \%$ of patients needed dialysis at presentation). As in other series of vasculitis with severe kidney involvement $[8,30]$, the clinical condition was poor at presentation; $25 \%$ of patients had BVAS $>21$. The lung was the second most affected organ; $31 \%$ of patients had severe hemorrhagic alveolitis. Moreover, there was a strong correlation between the severity of kidney and lung disease. The odds of severe hemorrhagic alveolitis were four times higher in patients needing dialysis at presentation. Although a high frequency of lung involvement in patients with severe kidney disease was observed in other series [7], the clinical significance of this association had not yet been underlined. It could be explained by the severity of vasculitis involving both organs, amplified by the consequences of severe uremia on capillary permeability favoring lung bleeding. MPO specificity was 3 times more frequent than PR3 specificity, lower than reported in the United Kingdom and Norway, but closer to reports from Spain, which supports a South to North gradient of MPO specificity distribution across Europe [33]. In this cohort with MPO-ANCA dominance and severe kidney disease, male gender and the need for dialysis at presentation were significantly associated with PR3-ANCA when serum creatinine was above $5 \mathrm{mg} / \mathrm{dL}$. This is in contrast with other studies which reported similar $[1,4,34]$ or worse [9] kidney disease at presentation in MPOANCA, not in PR3-ANCA. This discrepancy could be explained by the faster pre-admission deterioration of kidney function in PR3-ANCA [4] or by a combination of late referral with a selection bias, as patients were specifically referred because of severe kidney damage. On the other hand, the severity of kidney disease at presentation could have offset the phenotypic differences between MPO and PR3. However, our data suggest that in regions where MPO-ANCA incidence is high, the possibility of severe kidney disease in PR3-ANCA patients in a nephrology setting should be kept in mind.

The rate of complete remission was $60 \%$, lower than that reported in controlled trials (75-85\%) [35-37], where responsiveness was related mainly to the therapeutic protocols, but closer to that reported in observational studies (50-75 \%) [27, 30, 38, 39]. Possible explanations of the low rate of response are the severe clinical presentation and the infrequent use of plasmapheresis. In line with other cohorts of patients with severe kidney disease, in our patients the kidney failure described by the need for dialysis at presentation was an independent predictor of resistance to therapy while the ANCA pattern was not $[8,27,38]$. Thus, in a nephrology setting, the severity of kidney disease seems to overcome the prognostic significance of other factors. Interestingly, a higher platelets number was related to a positive response, as originally observed and interpreted by Westman [2] as reflecting a more severe immunological injury, not 
Table 5 Patients' characteristics according to outcome

\begin{tabular}{|c|c|c|c|}
\hline \multirow[t]{2}{*}{ Parameter } & \multicolumn{2}{|l|}{ Patients' death } & \multirow[t]{2}{*}{$P$ value } \\
\hline & No & Yes & \\
\hline Patients number & 51 & 24 & \\
\hline \multicolumn{4}{|l|}{ General } \\
\hline Gender (\% Male) & 53 & 38 & 0.21 \\
\hline Age (years) & $60[50 ; 68]$ & $63[60 ; 68]$ & 0.14 \\
\hline \multicolumn{4}{|l|}{ Vasculitis } \\
\hline MPO-ANCA (\%) & 65 & 35 & 0.31 \\
\hline PR3-ANCA (\%) & 78 & 22 & \\
\hline Severe lung hemorrhage (\%) & 29 & 39 & 0.39 \\
\hline BVAS & $15[14 ; 21]$ & $20[17 ; 21]$ & 0.01 \\
\hline $\begin{array}{l}\text { Response to induction } \\
\text { therapy }\end{array}$ & $76 \%$ & $25 \%$ & $<0.0001$ \\
\hline \multicolumn{4}{|l|}{ Inflammation } \\
\hline Hemoglobin (g/dL) & $8.6[7.7 ; 9.9]$ & $8.5[7.4 ; 9.8]$ & 0.79 \\
\hline WBC & $\begin{array}{l}9400[7600 ; \\
13900]\end{array}$ & $\begin{array}{l}10600[7800 ; \\
18400]\end{array}$ & 0.21 \\
\hline ESR & $100[78 ; 120]$ & $80[61 ; 120]$ & 0.20 \\
\hline Fibrinogen & $720[578 ; 820]$ & $645[525 ; 750]$ & 0.15 \\
\hline Serum albumin & $3.7[3.2 ; 4.0]$ & $3.2[2.8 ; 3.6]$ & 0.008 \\
\hline \multicolumn{4}{|l|}{ Kidney } \\
\hline \multicolumn{4}{|l|}{ Histopathologic class } \\
\hline Crescentic (\%) & 49 & 58 & 0.5 \\
\hline Focal (\%) & 4 & 8 & \\
\hline Mixed (\%) & 33 & 29 & \\
\hline Sclerotic (\%) & 14 & 4 & \\
\hline Serum creatinine $(\mathrm{mg} / \mathrm{dL})$ & $4.7[3.0 ; 7.4]$ & $6.9[4.1 ; 8.0]$ & 0.06 \\
\hline Proteinuria & $0.9[0.5 ; 2.2]$ & $0.8[0.6 ; 2.2]$ & 1.00 \\
\hline Hematuria & $230[180 ; 670]$ & $365[240 ; 1600]$ & 0.008 \\
\hline Dialysis at presentation (\%) & 22 & 38 & 0.15 \\
\hline
\end{tabular}

Values are median [quartile 1; quartile 3] if not otherwise specified PR3-ANCA patients positive for anti-proteinase 3 antibodies, MPO-ANCA patients positive for anti-myeloperoxidase antibodies, BVAS Birmingham Vasculitis Activity Score, WBC white blood cells, ESR erythrocite sedimentation rate

necessarily accompanied by more inflammation. This is also supported by our data showing higher albumin levels in responders and no difference in ESR or fibrinogen levels. Contrary to other series, older age was an indicator of a positive response. Possible explanations are a lower intensity of immune injury in elderly and increased disease awareness in elderly driving them to seek medical assistance in our particular population.

In spite of severe kidney disease at presentation, $51 \%$ of our patients were alive and dialysis free at the end of the observation period, which is similar to reports from studies enrolling vasculitis patients with severe kidney dysfunction (56-64 \%) [29, 40]. Moreover, the probability of kidney survival 5 years was comparable in our cohort to that reported in patients with similar kidney dysfunction at presentation [2, 29]. Unlike other studies where markers of kidney damage (serum creatinine [1, $2])$, urinary IgM [41] or ANCA pattern $[2,28]$ predicted ESRD, in our series the resistance to induction therapy and the need of dialysis at presentation were the independent predictors of kidney death. On the other hand, $27 \%$ of patients needing dialysis at presentation regained enough renal function to become dialysis-free at the end of the observation period. Accordingly, aggressive induction therapy is justified even in case of severe kidney failure. Simultaneously, it is to note that the severity of kidney status at presentation overwhelmed the severity of inflammation, the other organs lesions as well as ANCA specificity and the histopathological class as predictors of kidney death.

While kidney survival was associated with the initial organ damage, patient's survival depended on vasculitis activity at presentation as reflected by BVAS. Several studies reported associations between BVAS and long term outcome $[3,42]$. We observed a 6 times increase in risk of death for each three units rise in BVAS, which is in line with data reported in the largest multinational cohort of patients with ANCA-associated vasculitis where a small but significant effect of BVAS on survival was also found [3]. Thus, our data support the prognostic utility of BVAS and suggest a link between the initial organs damage and the long term prognosis, even when the clinical picture is dominated by the severity of kidney disease.

The other independent predictor of death was the response to induction therapy; the responders had five times more chances of survival than non-responders. As induction therapy was conducted according to current guidelines, including dose adjustment to renal function and age, this further underlines the limits of the existing therapeutic regimens.

Other studies reported differences in long term survival related to ANCA specificities [27, 28] while in our data neither kidney nor patient survival was related to ANCA serology. Nevertheless, in the previously mentioned EUVAS cohort, no ANCA-related dissimilarities in outcome were found and Flossmann et al. commented that previously reported differences in outcome could have been artifactual, caused by case-mix and underpowered adjustment for age and kidney damage [3]. Alternatively, when the consequences of vasculitis are severe - i.e. extensive kidney or other organs damage - they can conceal the initiating mechanism (ANCA specificity).

A histopathological classification of ANCA-associated glomerulonephritis was recently proposed by Berden et al. [19], aiming to improve prognosis of renal outcome and, eventually, to guide therapy. In our cohort, the proportions were similar in the case of the crescentic and 

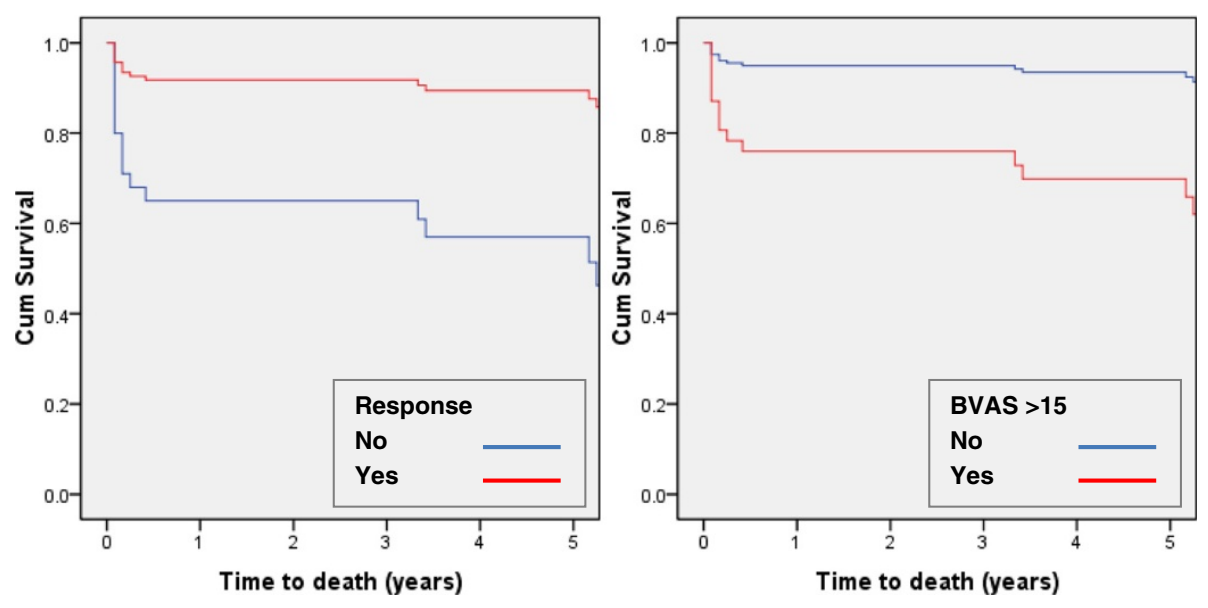

Fig. 3 Adjusted cumulative patient survival according to the response to induction therapy (left) and to BVAS (right). Legend: The median survival was 8.4 (7.1-9.6) years in responders vs. 4.7 (2.9-6.8) years in non-responders, $p<0.0001$ (left) and 7.5 (6.5-8.6) years in those with BVAS $\leq 15$ vs. 6.1 (4.5-7.6) years in those with BVAS $>15, p<0.0001$ (right). BVAS Birmingham vasculitis activity score ver 3

sclerotic classes (52 vs $55 \%$ and 11 vs $13 \%$ ), higher in the case of the mixed class (32 vs $16 \%$ ) and lower in the case of the focal class (5 vs $16 \%$ ) than reported by Berden et al. [19]. These differences could be due to a selection bias, as our patients were selected by the severity of kidney damage (which resulted in a lower frequency of focal class and a higher one in the mixed class), or to a limited reproducibility of the classification, as suggested by Ford et al. [20]. In our data, the histopathological classes were related neither to kidney function at presentation nor to kidney or patient outcome. However, our study had a low number of participants, which imposes caution in interpretation. Nevertheless, the validity of this classification was evaluated only in few studies so far and further research is clearly needed [20-23].

There are some limits of our study. The number of participants was low and the period of observation rather short, both limiting the statistical power. The single center design could be a limit for extrapolation of the results to other population, but data on Central East European population are scarce and on the other hand, this design increases the accuracy of data collection. ANCA were assessed by indirect immunofluorescence and/or by ELISA, and discrepancies between results obtained by these methods were described.

\section{Conclusions}

In ANCA positive pauci-immune crescentic glomerulonephritis, the kidney and patient outcomes are related to the severity of kidney damage at presentation and to the response to therapy, but not to ANCA specificity or to the histopathological class. Thus, "the blast" - the acute injury inducing severe organ damage - results in a peculiar distribution in histopathological classes, reduced responsiveness to therapy and poorer outcome, and could hide the prognostic influence of both "the trigger" - i.e. ANCA specificity - and of the histopathology lesions.

\section{Abbreviations}

MPO: Myeloperoxidase; PR3: Proteinase 3; ANCA: Anti-neutrophil cytoplasmic antibodies; BVAS: Birmingham Vasculitis Activity Score ver. 3; ESRD: End stage renal disease; ELISA: Enzyme-linked immunosorbent assay; Cl: Confidence interval; PHR: Proportional hazard ratios; HR: Hazard ratio; EUVAS: European vasculitis study group; CNS: Central nervous system; WBC: White blood cells; ESR: Erythrocyte sedimentation rate.

\section{Competing interests}

The authors declare that they have no competing interests.

\section{Authors' contributions}

IA, SS and GM participated in conception and design of the study, analysis and interpretation of data, drafting of the final article, AA and LT acquired the data. All authors read and approved the final manuscript.

\section{Acknowledgement}

We thank to Eugen Mandache, MD, PhD, for providing and for assistance with the kidney biopsies and to Liliana Bârsan, MD, for ANCA assay. These two colleagues had no financial support regarding this study.

Received: 11 October 2014 Accepted: 21 June 2015

Published online: 30 June 2015

\section{References}

1. Booth AD, Almond MK, Burns A, Ellis P, Gaskin G, Neild GH, et al. Outcome of ANCA-associated renal vasculitis: a 5-year retrospective study. Am J Kidney Dis. 2003;41(4):776-84.

2. Westman KW, Selga D, Isberg PE, Bladström A, Olsson H. High proteinase 3-anti-neutrophil cytoplasmic antibody (ANCA) level measured by the capture enzyme-linked immunosorbent assay method is associated with decreased patient survival in ANCA-associated vasculitis with renal involvement. J Am Soc Nephrol. 2003;14(11):2926-33.

3. Flossmann O, Berden A, de Groot K, Hagen C, Harper L, Heijl C, et al. Long-term patient survival in ANCA-associated vasculitis. Ann Rheum Dis. 2011;70(3):488-94.

4. Franssen CF, Gans RO, Arends B, Hageluken C, ter Wee PM, Gerlag PG, et al. Differences between anti-myeloperoxidase- and anti-proteinase 3-associated renal disease. Kidney Int. 1995;47(1):193-9.

5. Jennette JC, Falk RJ. Small-Vessel Vasculitis. N Engl J Med. 1997;337:1512-23. 
6. Fatma LB, El Ati Z, Lamia R, Aich DB, Madiha K, Wided S, et al. Alveolar hemorrhage and kidney disease: characteristics and therapy. Saudi J Kidney Dis Transpl. 2013;24(4):743-50.

7. Hruskova Z, Casian AL, Konopasek P, Svobodova B, Frausova D, Lanska V, et al. Long-term outcome of severe alveolar haemorrhage in ANCA-associated vasculitis: a retrospective cohort study. Scand J Rheumatol. 2013;42(3):211-4.

8. Lee T, Gasim A, Derebail VK, Chung Y, McGregor JG, Lionaki S, et al. Predictors of treatment outcomes in ANCA-associated vasculitis with severe kidney failure. Clin J Am Soc Nephrol. 2014;9(5):905-13.

9. de Joode AAE, Sanders JSF, Stegeman CA. Renal Survival in Proteinase 3 and Myeloperoxidase ANCA-Associated Systemic Vasculitis. Clin J Am Soc Nephrol. 2013;8:1709-17.

10. Millet A, Pederzoli-Ribeil M, Guillevin L, Witko-Sarsat V, Mouthon L. Antineutrophil cytoplasmic antibody-associated vasculitides: is it time to split up the group? Ann Rheum Dis. 2013;72(8):1273-9.

11. Fujimoto S, Watts RA, Kobayashi S, Suzuki K, Jayne DR, Scott DG, et al. Comparison of the epidemiology of anti-neutrophil cytoplasmic antibodyassociated vasculitis between Japan and the U.K. Rheumatology (Oxford). 2011;50(10):1916-20.

12. Ozaki S. ANCA-associated Vasculitis: Diagnostic and Therapeutic Strategy. Allergol Int. 2007:56:87-96.

13. Fujimoto S, Uezono S, Hisanaga S, Fukudome K, Kobayashi S, Suzuki K, et al. Incidence of ANCA-associated primary renal vasculitis in the Miyazaki Prefecture: the first population-based, retrospective, epidemiologic survey in Japan. Clin J Am Soc Nephrol. 2006;1(5):1016-22.

14. Gonzalez-Gay MA, Garcia-Porrua C, Guerrero J, Rodriguez-Ledo P, Llorca J. The epidemiology of the primary systemic vasculitides in northwest Spain: implications of the Chapel Hill Consensus Conference definitions. Arthritis Rheum. 2003:49(3):388-93.

15. Bajema IM, Hagen EC, Hermans J, Noël LH, Waldherr R, Ferrario F, et al. Kidney biopsy as a predictor for renal outcome in ANCA-associated necrotizing glomerulonephritis. Kidney Int. 1999;56:1751-8.

16. Vergunst $C E$, van Gurp $E$, Hagen EC, van Houwelingen HC, Hauer HA, Noël $\mathrm{LH}$, et al. An index for renal outcome in ANCA-associated glomerulonephritis. Am J Kidney Dis. 2003:41:532-8.

17. Bajema IM. Pathological classification of anti-neutrophil cytoplasmic antibody (ANCA)-associated glomerulonephritis. Clin Exp Immunol.

2011;164 Suppl 1:14-6.

18. de Lind van Wijngaarden RA, Hauer HA, Wolterbeek R, Jayne DR, Gaskin G, Rasmussen N, et al. Clinical and histologic determinants of renal outcome in ANCA-associated vasculitis: A prospective analysis of 100 patients with severe renal involvement. J Am Soc Nephrol. 2006;17(8):2264-74.

19. Berden E, Ferrario F, Hagen EC, Jayne DR, Jennette JC, Joh K, et al. Histopathologic classification of ANCA-associated glomerulonephritis. J Am Soc Nephrol. 2010;21(10):1628-36.

20. Ford SL, Polkinghorne KR, Longano A, Dowling J, Dayan S, Kerr PG, et al. Histopathologic and clinical predictors of kidney outcomes in ANCA-associated vasculitis. Am J Kidney Dis. 2014;63(2):227-35.

21. Nohr E, Girard L, James M, Benediktsson H. Validation of a histopathologic classification scheme for antineutrophil cytoplasmic antibody-associated glomerulonephritis. Hum Pathol. 2014;45(7):1423-9.

22. Tanna A, Guarino L, Tam FW, Rodriguez-Cubillo B, Levy JB, Cairns TD, et al. Long-term outcome of anti-neutrophil cytoplasm antibody-associated glomerulonephritis: evaluation of the international histological clasiffication and other prognostic factors. Nephrol Dial Transplant. 2014:0:1-8 [Epub ahead of print].

23. Ellis CL, Manno RL, Havill JP, Racusen LC, Duvuru G. Validation of the new classification of pauci-immune glomerulonephritis in a United States cohort and its correlation with renal outcome. BMC Nephrol. 2013;14:210.

24. Jennette JC, Thomas DB. Pauci-immune and Antineutrophil Cytoplasmic Antibody Mediated Crescentic Glomerulonephritis and Vasculitis. In: Jennette JC, editor. Heptinstall's Pathology of the kidney. 6th ed. Philadelphia: Lippincott Williams \& Wilkins; 2007. p. 644-58.

25. Mukhtyar C, Lee R, Brown D, Carruthers D, Dasgupta B, Dubey S, et al. Modification and validation of the Birmingham Vasculitis Activity Score (version 3). Ann Rheum Dis. 2009;68(12):1827-32.

26. Hellmich B, Flossmann O, Gross WL, Bacon P, Cohen-Tervaert JW, Guillevin $L$, et al. EULAR recommendations for conducting clinical studies and/or clinical trials in systemic vasculitis: focus on anti-neutrophil cytoplasm antibody-associated vasculitis. Ann Rheum Dis. 2007;66(5):605-17.
27. Harper L, Savage CO. ANCA-associated vasculitis at the end of the twentieth century - a disease of older patients. Rheumatology. 2005;44:495-501.

28. Weidner S, Geuss S, Hafezi-Rachti S, Wonka A, Rupprecht HD. ANCA-associated vasculitis with renal involvement: an outcome analysis. Nephrol Dial Transplant. 2004;19(6):1403-11.

29. Little MA, Nazar L, Farrington K. Outcome in glomerulonephritis due to systemic small vessel vasculitis: effect of functional status and non-vasculitic co-morbidity. Nephrol Dial Transplant. 2004;19(2):356-64.

30. Jayne DR, Gaskin G, Rasmussen N, Abramowicz D, Ferrario F, Guillevin L, et al. Randomized trial of plasma exchange or high-dosage methylprednisolone as adjunctive therapy for severe renal vasculitis. J Am Soc Nephrol. 2007;18(7):2180-8.

31. Watts RA, Mooney J, Skinner J, Scott DG, Macgregor AJ. The contrasting epidemiology of granulomatosis with polyangiitis (Wegener's) and microscopic polyangiitis. Rheumatology (Oxford). 2012;51:926-31.

32. Capizzi SA, Specks U. Does infection play a role in the pathogenesis of pulmonary vasculitis? Semin Respir Infect. 2003;18:17-22.

33. Watts RA, Lane SE, Scott DG, Koldingsnes W, Nossent H, Gonzalez-Gay MA, et al. Epidemiology of vasculitis in Europe. Ann Rheum Dis. 2001;60(12):1156-7.

34. Cohen BA, Clark WF. Pauci-immune renal vasculitis: Natural history, prognostic factors and impact of therapy. Am Journal Kidney Dis. 2000;36(5):914-24.

35. Pagnoux C, Mahr A, Hamidou MA, Boffa JJ, Ruivard M, Ducroix JP, et al. Azathioprine or methotrexate maintenance for ANCA-associated vasculitis. N Engl J Med. 2008;359(26):2790-803.

36. de Groot K, Harper L, Jayne DR, Flores Suarez LF, Gregorini G, Gross WL, et al. Pulse versus daily oral cyclophosphamide for induction of remission in antineutrophil cytoplasmic antibody-associated vasculitis: a randomized trial. Ann Intern Med. 2009;150(10):670-80

37. Jones RB, Tervaert JW, Hauser T, Luqmani R, Morgan MD, Peh CA, et al. Rituximab versus cyclophosphamide in ANCA-associated renal vasculitis. N Engl J Med. 2010;363(3):211-20.

38. Pagnoux C, Hogan SL, Chin H, Jennette JC, Falk RJ, Guillevin L, et al. Predictors of treatment resistance and relapse in antineutrophil cytoplasmic antibody-asociated small-vessel vasculitis. Comparison of two independent cohorts. Arthritis Rheum. 2008;58(9):2908-18.

39. Lionaki S, Blyth ER, Hogan SL, Hu Y, Senior BA, Jennette CE, et al. Classification of antineutrophil cytoplasmic autoantibody vasculitides: the role of antineutrophil cytoplasmic autoantibody specificity for myeloperoxidase or proteinase 3 in disease recognition and prognosis. Arthritis Rheum. 2012;64(10):3452-62.

40. Walsh M, Catapano F, Szpirt W, Thorlund K, Bruchfeld A, Guillevin L, et al. Plasma exchange for renal vasculitis and idiopathic rapidly progressive glomerulonephritis: a meta-analysis. Am J Kidney Dis. 2011;57:566-74.

41. Bakoush O, Segelmark M, Torffvit O, Ohlsson S, Tenoer J. Urine Ig M excretion predicts outcome in ANCA-associated renal vasculitis. Nephrol Dial Transplant. 2006;21(5):1263-9.

42. Gayraud M, Guillevin L, le Toumelin P, Cohen P, Lhote F, Casassus P, et al. Long-term follow-up of polyarteritis nodosa, microscopic polyangiitis, and Churg-Strauss syndrome: analysis of four prospective trials including 278 patients. Arthritis Rheum. 2001:44:666-75.

\section{Submit your next manuscript to BioMed Central and take full advantage of:}

- Convenient online submission

- Thorough peer review

- No space constraints or color figure charges

- Immediate publication on acceptance

- Inclusion in PubMed, CAS, Scopus and Google Scholar

- Research which is freely available for redistribution 